\title{
ARTICLE
}

\section{EGS5 simulations for the development of non-contacting system to online measure the radiotracer concentration in blood}

\author{
Azhar H. Malik ${ }^{*}$, Kenji Shimazoe and Hiroyuki Takahashi \\ School of Engineering, The University of Tokyo, 7-3-1 Hongo, Bunkyo-ku, Tokyo 113-8656, Japan
}

\begin{abstract}
Quantitative Positron Emission Tomography (PET) studies require the blood sampling which is very inconvenient and painful for the patients, handling of radioactive samples is risky to the workers and lengthy as well as less efficient to the imaging centers. Various approaches are proposed to reduce the exposure to the workers and number of blood samples to comfort the patients but no one fulfills all the requirements. We are designing a non-contacting system which can in-situ measure the radiotracer concentration in the blood and hence, provide Plasma Time-Activity Curve (PTAC). As a first step we consider a conventional system used to measure the dose in Nuclear Power Plant Systems (NPPS) with High Purity Germanium (HPGe) detector and a small animal PET system with Lutetium Aluminum Garnet with Avalanche Photo Diodes (LuAG-APD) pixelated detector blocks. Monte Carlo Electron Gamma Shower (EGS) 5 simulation tool is used to analyze the suitability of the systems and results are presented for a conventional parallel disc-detector system, a proposed perpendicular disc-detector system and a LuAG-APD animal PET tomograph. Although the conventional NPPS dose measurement system has the advantage of on-site measurement and best energy resolution but suffers from low detection efficiency and cannot be used in our case. However, the LuAG based PET tomograph with high detection efficiency, good spatial resolution, acceptable energy resolution and flexible arrangement to increase detection efficiency seems to be a more appropriate candidate.
\end{abstract}

Keywords: Monte Carlo EGS5 code; radiotracer concentration; PTAC; HPGe; absolute full-energy efficiency; LUAG-APD PET tomograph; sensitivity

\section{Introduction}

Quantitative PET studies may be used in non-invasive monitoring of drug pharmacology, estimation of glucose consumption in human and the estimate of tissue fractional uptake (and thus, regional metabolic rate of glucose) through dynamic PET data analyzed with mathematical modeling of Fluorodeoxyglucose $\left({ }^{18} \mathrm{FDG}\right)$ kinetics [1-2]. Among the different mathematical modeling approaches to model ${ }^{18} \mathrm{FDG}$ kinetics, compartmental modeling is most widely used for the uptake and clearance of radiotracer from tissue [3].

Mostly used three compartmental model was initially proposed by Sokoloff et al. and later extended by Huang et al. [4]. The model is based on few assumptions and using it alongwith PTAC, we can determine glucose consumption in human and the estimate of tissue fractional uptake (and thus, regional metabolic rate of glucose) [2].

Anyhow, kinetic modeling and dynamic acquisition along with blood sampling is the most accurate method for the PET quantitative studies [4]. However, the blood

*Corresponding author. Email: azhar2932@hotmail.com sampling is very inconvenient and painful for the patients, handling of radioactive samples is risky to the workers, and lengthy as well as less efficient for the imaging centers [2]. Various approaches like automated blood sampling and population based input functions are proposed to reduce the exposure to the workers and number of blood samples to comfort the patients. The first method is used for only first few minutes and then requires the manual sampling and later is not applicable in calculation of individual rate constants [5].

The blood is usually sampled from the peripheral artery or vein, according to blood sampling scheme, after few minutes of the injection of ${ }^{18} \mathrm{FDG}$ and many samples have to be drawn to obtain PTAC [2]. There are many other risks, like infection, in blood sampling alongwith the drawbacks mentioned above [6]. The activity in blood increases after injection, achieves peak in 3-5 min after injection and then decreases. After 20 min of injection, it becomes almost constant around $740 \mathrm{kBq} / \mathrm{ml}$ [2] and thus we can assume artery as cylindrical source of almost constant activity. We can in-situ measure the radiotracer concentration in blood by measuring the vascular activity and avoid problems which arise due to the manual blood sampling. 
We are developing a non-contacting system which can in-situ measure the radiotracer concentration in the blood and is capable to provide PTAC, input function for almost all quantitative PET studies, to fulfill all the requirements. We consider a conventional system used to on-site measure the dose in NPPS with HPGe detector and a small animal PET like system with LuAG-APD pixelated detectors. Previously we compared our EGS5 simulation results for a point source and a parallel disc source with GEANT4 and MCNP simulation, and experimental results and a good agreement was found [7]. In this paper, we mainly focused on our proposed perpendicular disc detector system and analyzed the suitability of the proposed system for designing an in-situ blood activity measurement system.

\section{EGS5 simulation results}

\subsection{HPGe dose measurement system}

The blood vessel is assumed to contain the radionucei ${ }^{18} \mathrm{~F}$ which emits positron whose annihilation with electron results in the emission of two $511 \mathrm{keV}$ photons in opposite directions. The detector, single or in form of a ring like PET system around the blood vessel, measures the activity in the blood which corresponds to the concentration of radio-tracer in the blood. Source strength is given by the following relation [8],

$$
S=N_{\mathrm{det}} \frac{4 \pi}{\varepsilon_{i p} \Omega}
$$

where, $N_{\text {det }}$ is the number of gamma rays detected by detector under full energy peak, $S$ is the radiation quanta emitted by source over measurement period, $\varepsilon_{\text {ip }}$ is intrinsic peak efficiency and $\Omega$ is the solid angle in steradians. Analytical and approximated relations exist to determine the solid angle subtended by detector at source position for a point source and a parallel disc source as described in detail in [8-9]. In our case, the source is not a point source but instead it is cylindrical source which will be modeled by considering the various parameters which include the dimensions of blood vessel, its thickness, the blood velocity, the medium and distance between the source and detector etc. As a first and simpler case, we discretized, as shown in Figure 1 the cylindrical source into small discs of negligible thickness and found the absolute full-energy efficiency (the ratio of number of photons detected with energy $E$ in photopeak to the total number of photons emitted by source with same energy [8]) for perpendicular disc detector system at various radial positions and later used to find the expected counts from the cylindrical source.

\subsubsection{Point source}

The point source is the simplest case and analytical relationship exists for the calculation of solid angle when point source is placed along the axis of right cylindrical detector. Thus, we can obtain efficiency by using Eq. (1) for various values of gamma-ray energies.

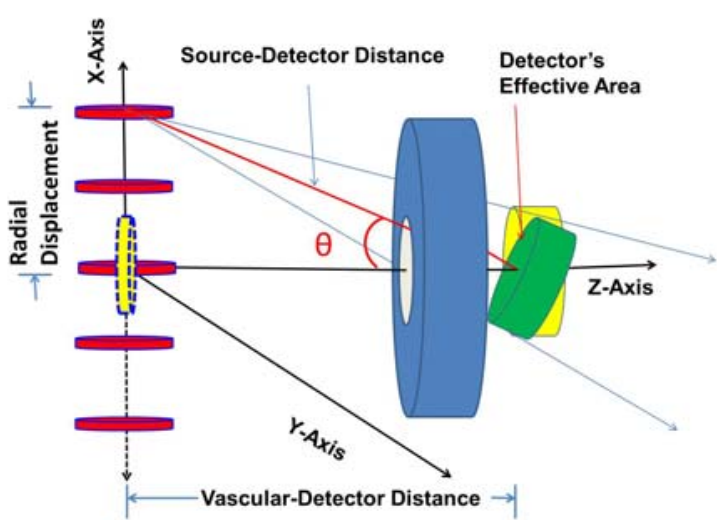

Figure 1. A perpendicular disc-detector system with the discs at different positions as result of cylindrical source discretization and a parallel disc detector system (only 1 dotted disc with center on detector axis is shown).

of practical interest [10].

We placed point source at different radial positions and the source biasing technique is used to simulate absolute full-energy efficiency. The results are compared with those obtained by Rodenas et al. by using MCNP as well as experiment [10] and Rehman et al. by using GEANT4 [11]. The difference between our results of a point source at center and those obtained earlier was less than $9 \%$ except the case where it was $12.3 \%$ with MCNP for $20 \mathrm{~mm}$ collimator diameter (coll. dia.) and $1332 \mathrm{keV}$, see [7] for detail. After the verification of simulation model, through comparison of EGS5 simulation results of a point source at center with those of GEANT4 and MCNP, the absolute full-energy efficiency versus radial distance curves for energies $662-1332 \mathrm{keV}$ alongwith $511 \mathrm{keV}$, energy of interest in this study, are obtained and are shown in Figure 2.

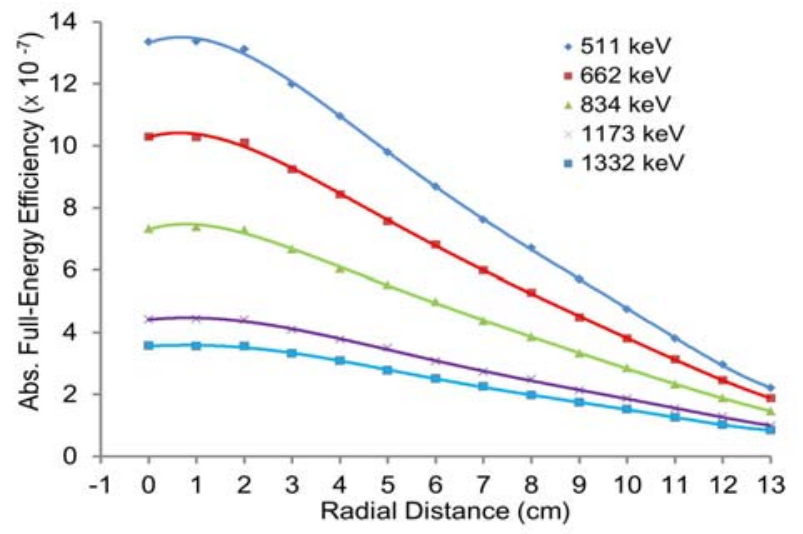

Figure 2. Variation of abs. full-energy efficiency with radial distance of point source for coll. dia. of $20 \mathrm{~mm}$.

\subsubsection{Parallel disc detector system}

A disc source is considered with center along detector axis as a parallel disc detector system. For such system, the absolute full-energy efficiency can be found by using the Eq. (1) with the difference of calculation of the effective solid angle. In order to find solid angle of disc source for parallel disc detector system, Ruby's formula is used which is quite complicated and approximated algebraic expressions or full series expansion formulas 
may be used as described in great detail in [9]. These analytical formulas may be used as bench mark to find the solid angle and verify the Monte-Carlo technique's validity which may further be extended to find solid angles of irregular and complex geometries. The source radius is varied from zero (point source) to radius of 5 $\mathrm{cm}$ and results are compared with those of GEANT4 [11]. The variation of absolute full-energy efficiency with source radius for various energies is shown in Figure 3. The behavior is in a good agreement with the point source and already published results of GEANT4.

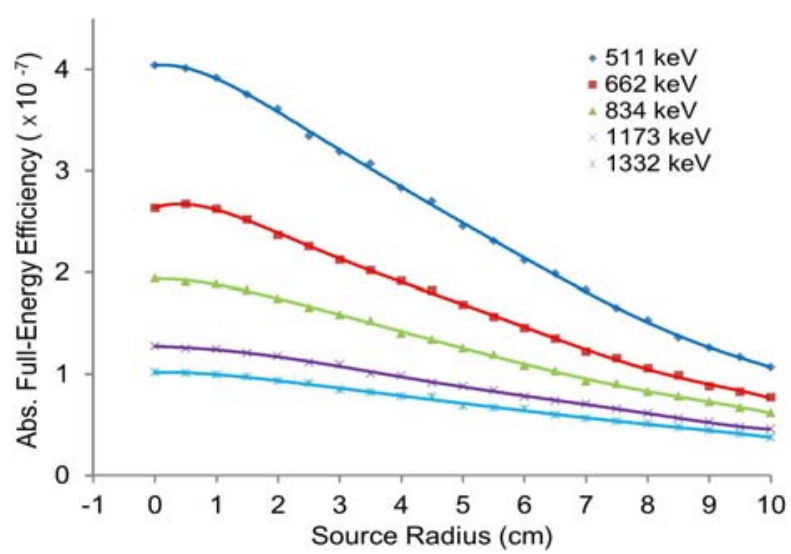

Figure 3. Abs. full-energy efficiency versus source radius for parallel disc-detector system for $511-1332 \mathrm{keV}$ energies. Disc is $1 \mathrm{~m}$ away from detector face and coll. dia. is $10 \mathrm{~mm}$.

\subsubsection{Perpendicular disc detector system}

As described previously, the results are verified for a point source and a parallel disc source. The disc is rotated, see Figure 1, and the system dimensions are reduced which suit present case. For the vasculardetector distance of $10 \mathrm{~cm}$, collimator size of $10 \mathrm{~mm}$ and $511 \mathrm{keV}$ energy, the variation of full-energy efficiency is studied for different source radii and radial positions. Initially, the disc is rotated and the variation of absolute full-energy efficiency is determined at various angles for different source radii. As clear from Figure 4, the absolute full-energy efficiency is constant with angle of rotation for point source which is consistent with the expected behavior. For $90^{\circ}$ angle of rotation, the parallel disc detector system becomes parallel disc detector system and the values are consistent with as expected on the basis of previous section. Hence, we obtained the $511 \mathrm{keV}$ absolute efficiency curve with radial distance for a perpendicular disc detector case and is shown in Figure 5 alongwith $662 \mathrm{keV}$ for the sake of comparison.

Finally we discretized the blood vascular and considered the discs at different radial positions that form the cylindrical blood vascular. We assumed the typical values of radioactivity injected in body for PET and uniform distribution in the blood. The blood vascular with the typical length of $20 \mathrm{~cm}$ and diameter of $2.35 \mathrm{~mm}$ is divided into 41 discs and the expected counts obtained in $30 \mathrm{~min}$ measurement time, by using the absolute full-energy efficiency values, were 1652 which shows that system is not suitable in this case.

\subsection{Compact LUAG-APD based animal PET}

Recently a compact LuAG-APD based animal PET tomograph is developed in our laboratory with spatial resolution $1.76 \mathrm{~mm}$ by using ${ }^{22} \mathrm{Na}$ columnar source [12]. EGS5 simulations of 4 block detectors with point source at center give the sensitivity of $0.83 \mathrm{cps} / \mathrm{kBq}$ which shows promise for measurement of activity in the blood vascular. Inclusion of 8 block detectors to increase the sensitivity and variable crystal-to-crystal distance makes this system to be considered for present application.

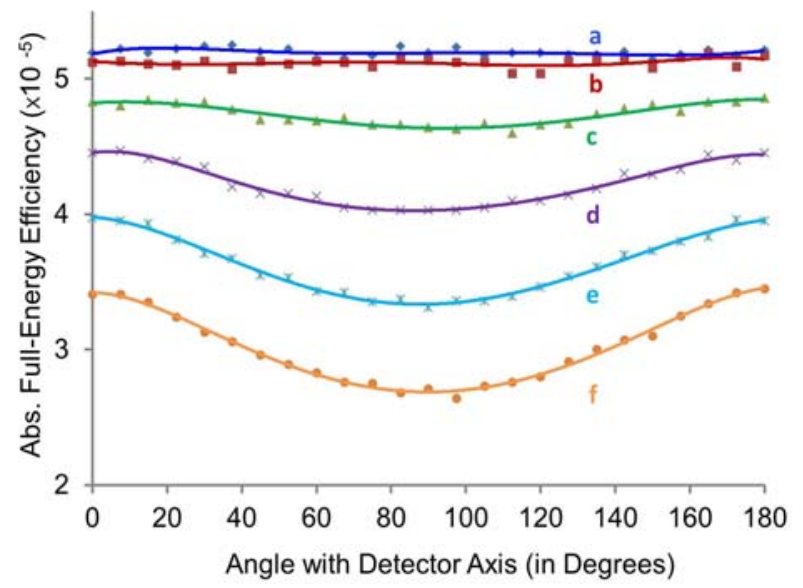

Figure 4. Abs. full-energy efficiency versus angle of rotation of disc with detector axis, $\left(90^{\circ}\right.$ parallel disc-det.), for (a) point source, (b) 0.2 , (c) 1 , (d) 2, (d) 3, and (f) $4 \mathrm{~cm}$ 's disc radius for $511 \mathrm{keV}$ incident gamma ray energy and $10 \mathrm{~mm}$ coll. dia.

\section{Discussion}

The simulation results of a point source and a disc source are presented for two collimator diameters and five gamma-ray energies of interest. In first part, the point source is placed at fix distance of $1 \mathrm{~m}$ and moved in radial direction. As shown in Figure 2, the absolute efficiency remains almost same as long as the radial distance of the point source is less than the collimator radius and then it starts decreasing. This is due to the reason that the thickness of lead between source and detector increases with the radial distance and the attenuation of gamma-rays increases in lead collimator. The results are compared with those, obtained earlier by using codes MCNP and GEANT4 respectively as well as experimentally and a good agreement is found.

In the case of the disc source, there are two arrangements. One is parallel disc detector system and the other one is perpendicular disc detector system. For first arrangement, a disc source is placed at a distance of $1 \mathrm{~m}$ and has the center on axis of cylindrical detector. The radius of the source is varied from zero (point source) to $10 \mathrm{~cm}$ and again the absolute full-energy efficiency is measured for 10 and $20 \mathrm{~mm}$ collimator diameters for above mentioned energies. The results are compared with literature values and a good agreement is found. The trend of the absolute full-energy efficiency is decreasing with the increase in radius. The disk source, with negligible thickness, was assumed to have same 
total activity uniformly distributed on whole surface whereas, the specific planar activity decreases with increase in radius. The absolute full-energy efficiency remains almost constant till the radius of source is comparable to collimator radius and after that the absolute full-energy efficiency decreases with increasing the source radius as shown in Figure 3.

Finally the disc is placed perpendicular to the axis of detector and again absolute efficiency at different radial position for various source radii is determined and an increase in the difference between two arrangements is observed with the increase in source radius. We assumed the typical values of radioactivity injected in body for PET and concluded that system is not suitable to measure the activity of such weak sources. However, the LuAG-APD pixelated detectors system with high detection efficiency, good spatial resolution, acceptable energy resolution and flexible arrangement seems to be an appropriate candidate. The system is under development and initial experiments will be performed by using a point source to verify simulation results prior to phantom studies.

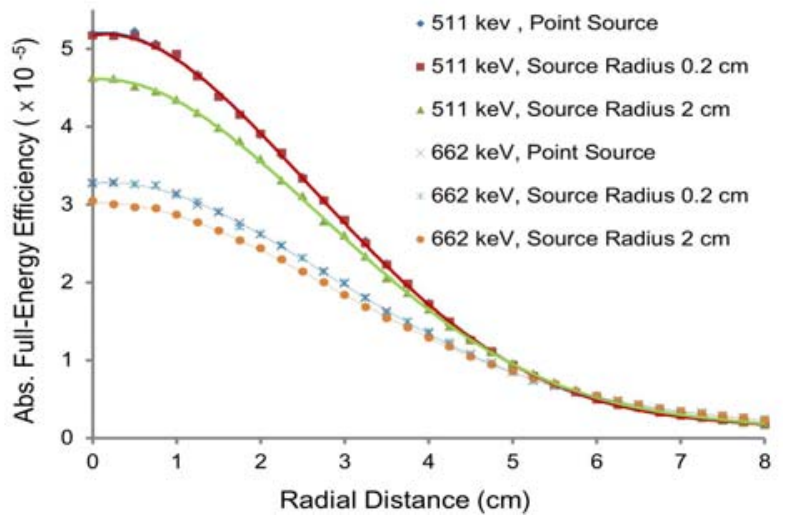

Figure 5. Abs. full-energy efficiency versus radial distance of perpendicular disc of different radii and 511 and $662 \mathrm{keV}$ gamma energies. The disc is $10 \mathrm{~cm}$ away from detector face.

\section{Conclusions}

EGS5 simulation results for energy range 662-1332 $\mathrm{keV}$ with those obtained experimentally as well as by using other Monte Carlo codes MCNP and GEANT4 are compared. We checked the feasibility of our proposed perpendicular disc-detector system for the measurement of activity of blood. Although the NPPS dose measurement system has the advantage of best energy resolution but suffers low detection efficiency and cannot be used for the activity measurement of such weak sources in reasonable measurement time interval.

\section{Acknowledgements}

The author is thankful to Monbukagakusho (MEXT), Japan for funding PhD study under ID: 103187.

\section{References}

[1] R. Myers, The biological application of small animal PET imaging, Nucl. Med. Bio. 28 (2001), pp. $585-593$.

[2] D. Feng, K. P. Wong, C. M. Wu and W. C. Siu, A technique for extracting physiological parameters and the required input function simultaneously from PET image measurements: theory and simulation study, IEEE Trans. on Inform. Tech. in Biomed. 1 (1997), pp. 243-254.

[3] P. E. Valk, D. L. Bailey, D. W. Townsend and M. N. Maisey, Positron Emission Tomography: Basic Science and Clinical Practice, Springer, London, (2003), pp. 150-151, ISBN 1-85233-485-1.

[4] K. Wienhard, Measurement of glucose consumption using [18F]fluorodeoxyglucose, Methods. 27 (2002), pp. 218-25.

[5] Stefan Eberl, Ali R. Anayat, Roger R. Fulton, P. K. Hooper and Michel J. Fulham, Yamamoto, Evaluation of two population-based input functions for quantitative neurological FDG PET studies, Eur. J. Nucl. Med. 24 (1997), pp. 299-304.

[6] K. Chen, D. Bandy, E. Reiman, S.C. Huang, M. Lawson, D. Feng, L.S. Yun and A. Palant, Noninvasive quantification of the cerebral metabolic rate for glucose using positron emission tomography, ${ }^{18} \mathrm{FDG}$, the Patlak method, and an image derived input function, J. Cereb. Blood Flow Metab. 18 (1998), pp. 716-723.

[7] Azhar H. Malik, Y. Nakamura, K. Shimazoe and H. Takahashi, Design of a non-contacting system to measure the radiotracer concentration in blood for nuclear medicine applications, Proc. $26^{\text {th }} \mathrm{KEK}$ Workshop on Radiation Detectors and Their Uses, Tsukuba, Japan, Jan. 24-25, 2012.(In Press)

[8] G.F. Knoll, Radiation Detection and Measurement, John Wiley \& Sons, New York, (2000), pp. 150-151, ISBN 978-0-471-07338-3.

[9] S. Pomme, A complete series expansion of Ruby's solid-angle formula, Nucl. Instr. Meth. in Phy. Res. A. 531 (2004), pp. 616-620.

[10] J. Rodenas, A. Martinavarro and V. Rius, Validation of the MCNP code for the simulation of Ge-detector calibration, Nucl. Instr. Meth. in Phy. Res. A. 450 (2000), pp. 88-97.

[11] S. U. Rehman, S. M. Mirza, N. M. Mirza and M. T. Siddique, GEANT4 simulation of photo-peak efficiency of small high purity germanium detectors for nuclear power plant applications, Annal. Nucl. Ener., 38 (2011), pp. 112-117.

[12] K. Shimazoe, Y. Wang, H. Takahashi, K. Kamada, M. Yoshino, J. Kataoka, Y. Yamaya, T. Yanagida, A. Yoshikawa and K. Kumagai, Time over threshold based digital animal PET (TODPET), IEEE Nucl. Sci. Symp. Conf. Rec., MIC15.S-44, (2011), pp. 3267-3271. 